\title{
A historical literature review on the role of posterior axillary boost field in the axillary lymph node coverage and development of lymphedema following regional nodal irradiation in breast cancer
}

\author{
Mahdieh Afkhami Ardekani ${ }^{1}{ }^{1}$, Hamed Ghaffari $\circledast^{2}$, Afrouz Mardi ${ }^{\circledR}{ }^{3}$, Soheila Refahi ${ }^{4}$ \\ ${ }^{1}$ Department of Radiology, Faculty of Para-Medicine, Hormozgan University of Medical Sciences, Bandar-Abbas, Iran \\ ${ }^{2}$ Department of Medical Physics, School of Medicine, Iran University of Medical Sciences, Tehran, Iran \\ ${ }^{3}$ Department of Public Health, School of Health, Ardabil University of Medical Sciences, Ardabil, Iran \\ ${ }^{4}$ Department of Medical Physics, Faculty of Medicine, Ardabil University of Medical Sciences, Ardabil, Iran
}

\begin{abstract}
To elucidate whether (1) a posterior axillary boost (PAB) field is an optimal method to target axillary lymph nodes (LNs); and (2) the addition of a PAB increases the incidence of lymphedema, a systematic review was undertaken. A literature search was performed in the PubMed database. A total of 16 studies were evaluated. There were no randomized studies. Seven articles have investigated dosimetric aspects of a PAB. The remaining 9 articles have determined the effect of a PAB field on the risk of lymphedema. Only 2 of 9 articles have prospectively reported the impact of a PAB on the risk of lymphedema development. There are conflicting reports on the necessity of a PAB. The PAB field provides a good coverage of level I/II axillary LNs because these nodes are usually at a greater depth. The main concern regarding a PAB is that it produces a hot spot in the anterior region of the axilla. Planning studies optimized a traditional PAB field. Prospective studies and the vast majority of retrospective studies have reported the use of a PAB field does not result in increasing the risk of lymphedema development over supraclavicular-only field. The controversies in the incidence of lymphedema suggest that field design may be more important than field arrangement. A key factor regarding the use of a PAB is the depth of axillary LNs. The PAB field should not be used unless there is an absolute indication for its application. Clinicians should weigh lymphedema risk in individual patients against the limited benefit of a $P A B$, in particular after axillary dissection. The testing of the inclusion of upper arm lymphatics in the regional LN irradiation target volume, and universal methodology measuring lymphedema are all areas for possible future studies.
\end{abstract}

Key words: posterior axillary boost; breast cancer radiotherapy; axillary lymph node; regional nodal irradiation Rep Pract Oncol Radiother 2021;26(4):635-646

\section{Introduction}

The radiotherapeutic management of the regional lymph nodes (RLNs) for breast cancer patients remains important, but it is technically challenging.
Traditional approach to treat supraclavicular (SC) fossa and axilla is the use of an anterior-posterior (AP, i.e., anterior SC fossa field) and posterior axillary boost $(\mathrm{PAB})$ field arrangement $[1-3]$. In other words, the majority of breast cancer patients with

Address for correspondence: Hamed Ghaffari, Department of Medical Physics, School of Medicine, Iran University of Medical Sciences, Tehran, Iran, tel: +98911 8555899; e-mail: hamedghaffari@yahoo.com 
nodal involvement were treated with SC field alone or in combination with a PAB field. The goal of a PAB field arrangement is to ensure complete dose coverage of SC fossa and levels I/II/III axilla [3]. The majority of dose is delivered through a SC field prescribed to the depth of maximum dose or $3-\mathrm{cm}$ depth to cover SC fossa and level III axilla [3]. However, more lateral level I/II axilla have a deeper location in comparison with the SC nodes. Therefore, sufficient dose may not be delivered to these regions. To ensure adequate coverage of level I/II axilla a PAB field is used.

Nevertheless, there are considerable debates regarding the effectiveness of a PAB field. To date, several studies have investigated dosimetric aspects of the PAB field and reported that it may not be an optimal technique to treat level I/II axillary LNs, in particular in the new era of breast cancer radiotherapy (RT) [1-4]. In addition, the effect of a PAB on lymphedema development in breast cancer patients remains incompletely understood. Lymphedema has an important effect on patient's quality of life (QOL) $[5,6]$. Taken together, it is, therefore, important for radiation oncologists to clearly understand dosimetric and clinical aspects of a PAB field.

Therefore, the objectives of this literature review were (1) to evaluate whether a PAB field is an optimal method to target SC and axillary LNs; and
(2) to investigate whether the addition of a PAB increases the incidence of lymphedema.

\section{Materials and methods}

\section{Search strategy and selection criteria}

PubMed database was used for the literature searching from January 1990 to December 2019 using the following search terms: (Breast cancer radiotherapy) AND ("Posterior axillary boost" OR "Regional nodal irradiation" OR "Regional radiotherapy"). The following selection criteria were adopted:

- inclusion criteria: studies on human, specifically investigating the dosimetric and clinical aspects or clinical efficacy of a PAB, full-text published articles in English;

- exclusion criteria: review articles, animal studies, lack of relevant outcome data, non-English written articles, editorials, commentaries, conference abstracts.

To select a full-text article for review, search results were screened based on title and abstract (Fig. 1). Outcomes were expressed as reported originally. However, meta-analysis and statistical analysis were not carried out because of the obvious heterogeneity of patient cohort in the selected articles and variation in the outcomes reported.

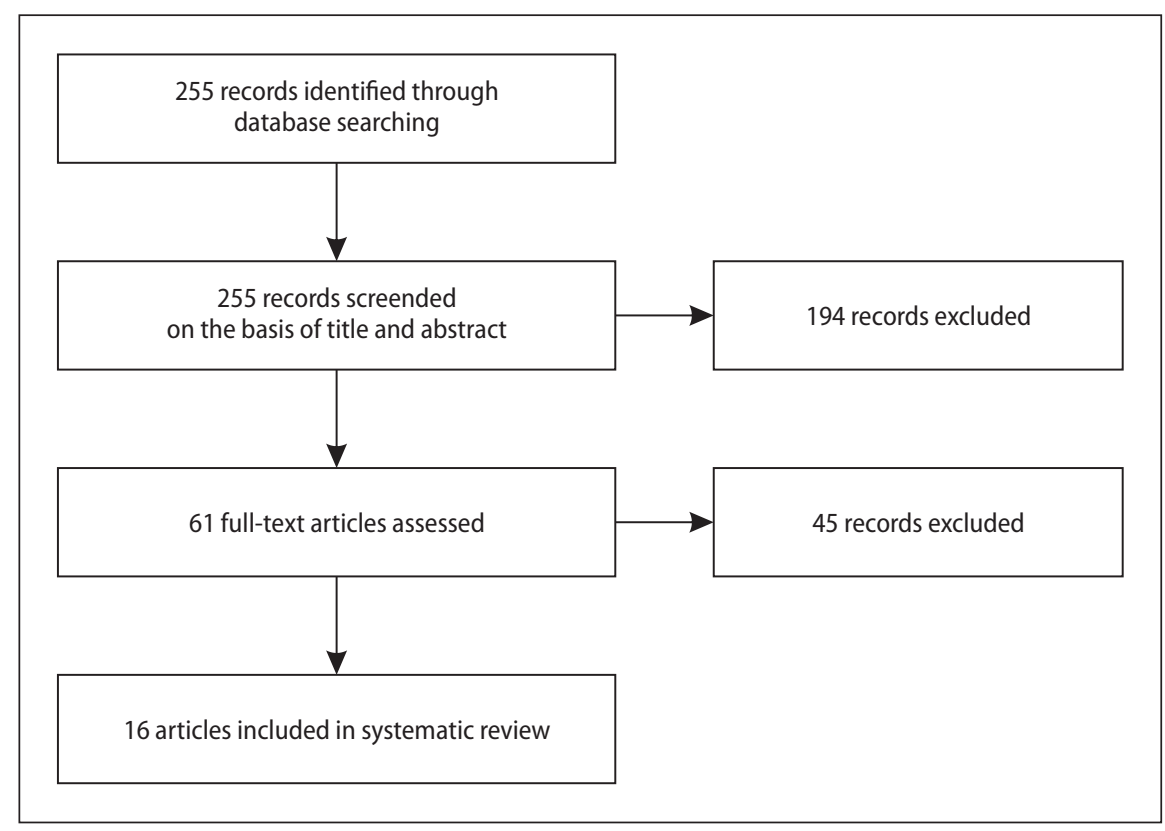

Figure 1. Flowchart for literature search 


\section{Results and discussion}

\section{Study selection and study characteristics}

Two-hundred and fifty-five potentially relevant articles were identified and screened for title and abstract. Sixty-one full-text articles were evaluated for eligibility. Of these, 45 articles were excluded after full-text reading. Finally, a total of 16 articles that met the inclusion criteria were included in this literature review, as displayed in Figure 1. Seven articles have investigated dosimetric aspects of a PAB. The remaining 9 articles have determined whether the addition of a PAB field increases the risk of lymphedema. There were no randomized studies. Only 2 of 9 articles have prospectively reported the impact of a PAB on the risk of lymphedema development. Table 1 and 2 provide a summary of the characteristics of the included studies and their main outcomes.

\section{Posterior axillary boost field}

Historically, a PAB field was developed based on the fact that the axillary LNs are deeper than the SC LNs in the pre-computed tomography (CT) era [1]. On the other hand, if a SC field provides inadequate coverage of deep LNs owing to axillary node separation, then a PAB field may be used to assure optimal dose distribution in the mid-axillary area [3]. In some centers, a PAB field was added if there were gross extranodal spread of tumor, inadequate axillary dissection, positive axillary nodes, and axillary failure without previous history of irradiation $[3,8,13]$. The medial border of a PAB field is typically defined by 1.5 to $2 \mathrm{~cm}$ of the lung. The lateral border is at the posterior axillary fold; however, the posterior axillary fold is not a useful plain film or CT landmark. The inferior border will match the breast tangentials and superior border splits the clavicle. Also, the gantry may be tilted 10-15 degrees to spare the trachea, esophagus, and spinal cord. Of note, if the medial PAB border is defined by $2 \mathrm{~cm}$ of lung, there is no need for a gantry tilt. The prescribed dose is such that the combination dose distribution ensures adequate coverage of nodal volumes. There is an overlap between the anterior SC field and the PAB field with the amount of overlap varying from one patient to another. The borders of a PAB field may be edited to block hot spots owing to overlap with a SC field.

\section{Dosimetric effect of posterior axillary boost field}

Table 1 summarizes studies on dosimetric aspects of the PAB field in axillary LNs irradiation. A deep understanding about the depths of the SC and axillary LNs is an important step to elucidate the necessity of a PAB. Studies have reported that the depth of the SC and axillary LNs varies widely $[1,8]$. In a study based on CT data, Bentel et al. measured the maximum depth of the SC and axillary LNs in 49 patients at the Duke University of Medical Center [1]. Furthermore, these authors determined the relationship between the SC and axillary LN depth and patient's size represented by the AP diameter. The median of the SC LNs depth was $4.3 \mathrm{~cm}$ in a range from 2.4 to $9.5 \mathrm{~cm}$. The depth of the axillary LNs ranged from 1.4 to $8 \mathrm{~cm}$, with a median of $4.3 \mathrm{~cm}$. It was observed that the axillary LNs lie at approximately the same depth or shallower than the SC in most patients. The results of that study demonstrated that the dose to the axillary LNs was within $\pm 5 \%$ of the SC dose in $53 \%$ of patients and was $90 \%$ or more of the dose delivered in the SC in $90 \%$ of patients, when an anterior 6-MV beam only was employed to treat both the SC and axillary LNs. Therefore, they concluded that these results can obviate the need for a PAB field. As suggested by Bentel et al., the use of higher energy beams, an anterior beam only, or opposed fields may be reasonable when the SC and axillary LNs are deep [1]. Furthermore, a deeper prescription dose point can provide sufficient planning target volume (PTV) coverage for patients with deep nodal volumes, this, however, results in more intense hot spots. A concern regarding higher energy beam is potential underdosing of superficial LNs. When a high energy beam is employed, the placement of bolus on the SC region is necessary owing to the behavior of depth dose. Depth dose does not decrease in a linear fashion. As a result, the use of an anterior beam only is suboptimal in some cases. However, the anterior beam may be useful for selected patients with small separations. It is worthwhile to mention that patients with elevated separations that require high energy beams do not have superficial LNs.

In another study, Goodman et al. measured the depths of SC and level I-III axillary LNs relative to the anterior skin surface [8]. The median (range) depths of the SC and level I-III axillary LNs were 3.7 


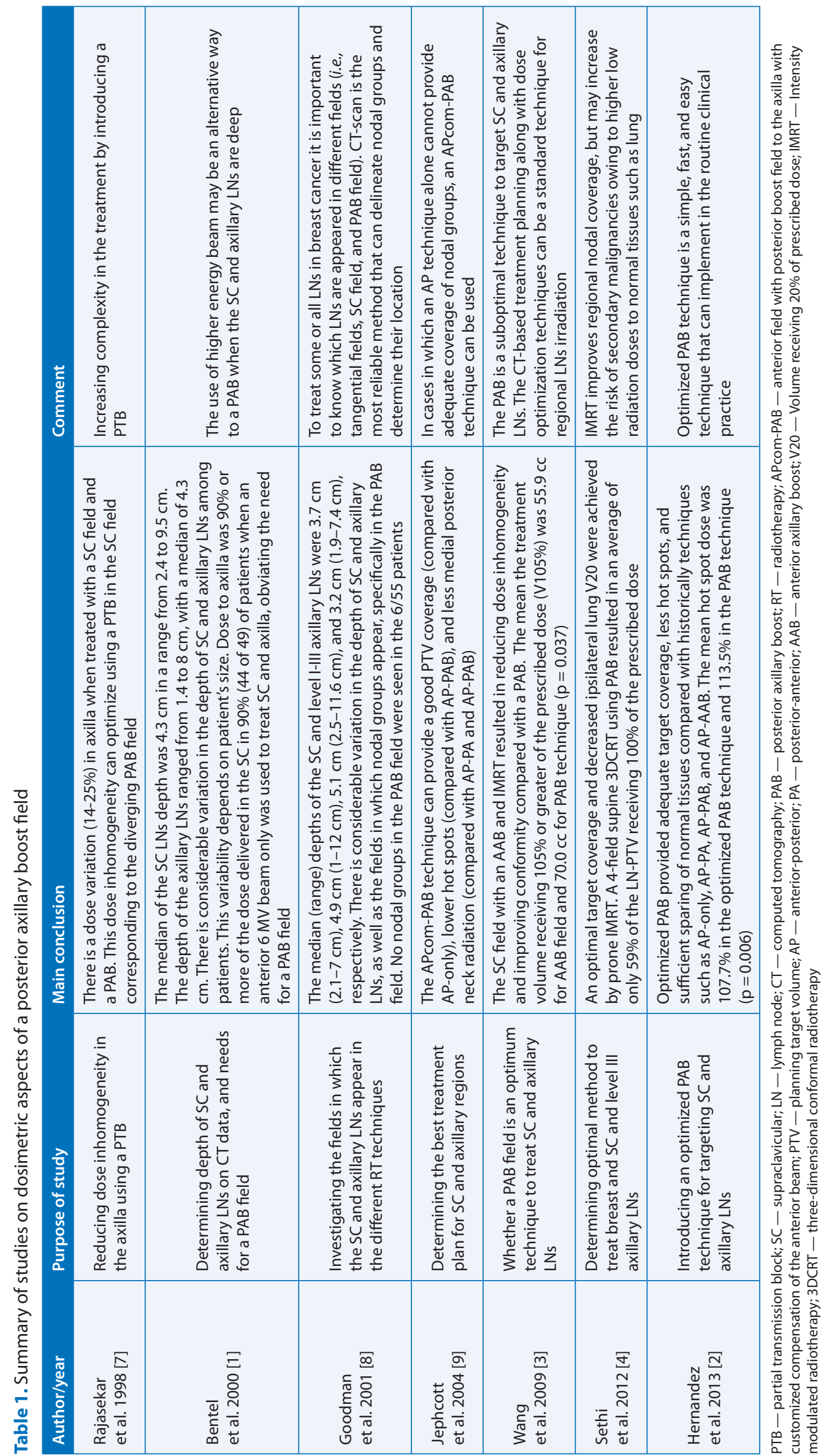




\begin{tabular}{|c|c|c|c|c|c|c|c|c|}
\hline $\begin{array}{l}\text { 荘 } \\
\frac{0}{0} \\
\frac{.0}{x}\end{array}$ & 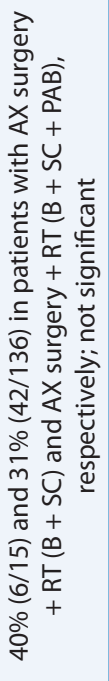 & 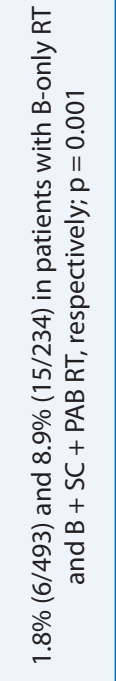 & 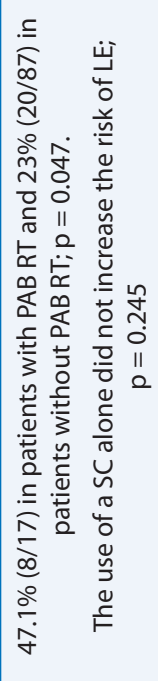 & 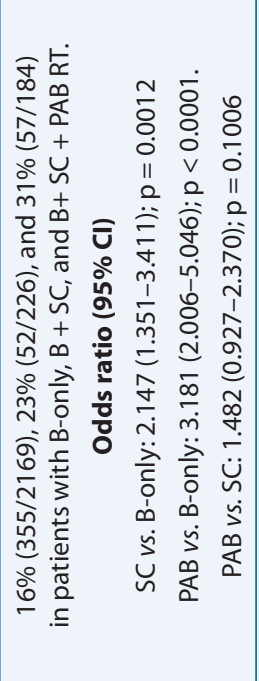 & 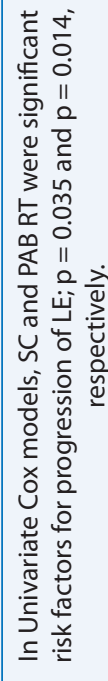 & 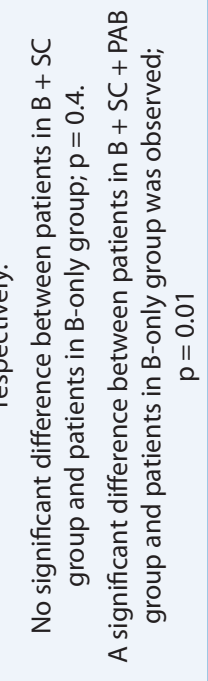 & 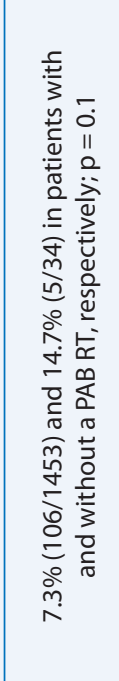 & 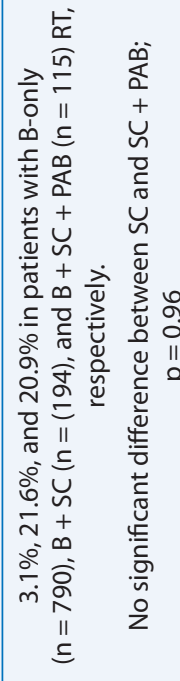 \\
\hline 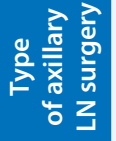 & 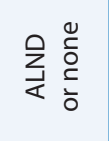 & 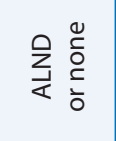 & $\sum_{\frac{1}{<}}$ & 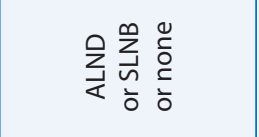 & & 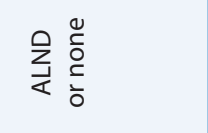 & 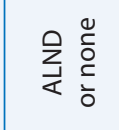 & 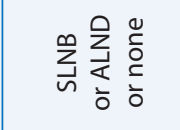 \\
\hline 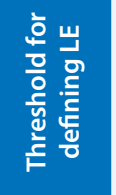 & $\underset{\wedge}{E}$ & $\underset{N}{E}$ & 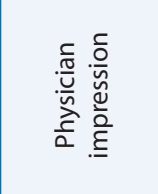 & 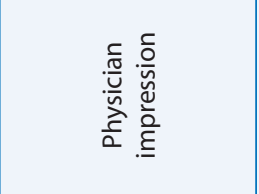 & & $\underset{N}{E}$ & 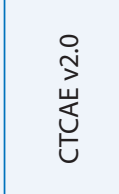 & 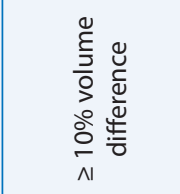 \\
\hline 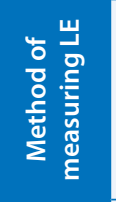 & 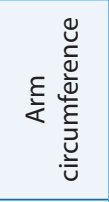 & 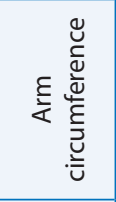 & 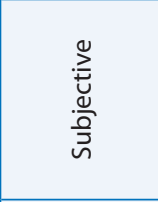 & 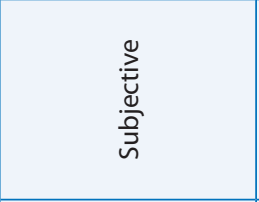 & & 妾 & 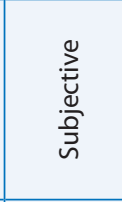 & 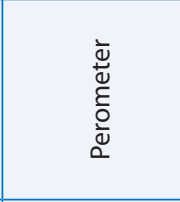 \\
\hline 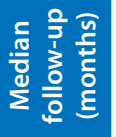 & $\infty$ & के & $\stackrel{\mathbb{d}}{d}$ & $\bar{\infty}$ & & i & $\stackrel{\propto}{z}$ & 节 \\
\hline $\begin{array}{l}\text { to } \\
\stackrel{\nu}{\Sigma}\end{array}$ & 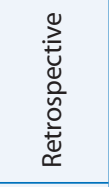 & 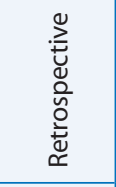 & 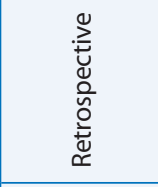 & 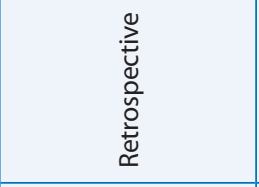 & & 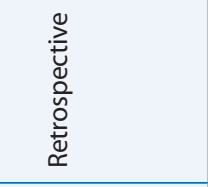 & 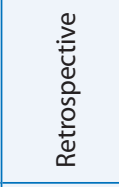 & 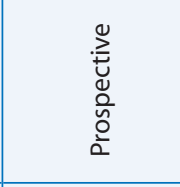 \\
\hline 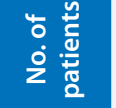 & $\stackrel{\stackrel{\infty}{\varrho}}{=}$ & $\stackrel{\aleph}{\wedge}$ & $\stackrel{\Perp}{\subseteq}$ & 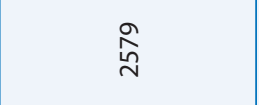 & & $\stackrel{\bullet}{\sim}$ & g্ & ڤે \\
\hline 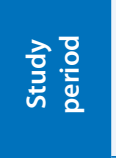 & $\begin{array}{l}\text { ò } \\
\sigma \\
\vdots \\
\alpha \\
\alpha \\
\sigma\end{array}$ & $\begin{array}{l}\text { na } \\
\sigma \\
1 \\
\alpha \\
\alpha \\
\sigma\end{array}$ & 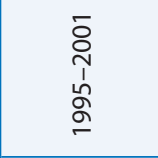 & 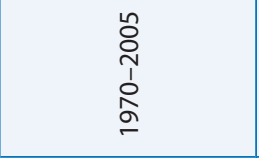 & & 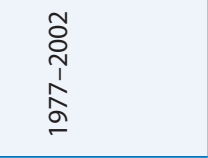 & 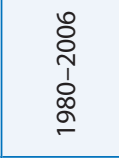 & 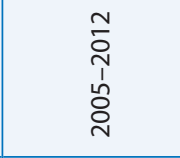 \\
\hline 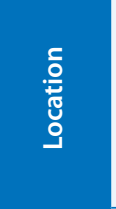 & 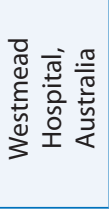 & 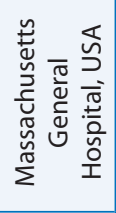 & 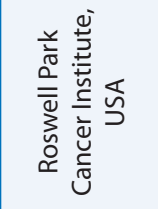 & 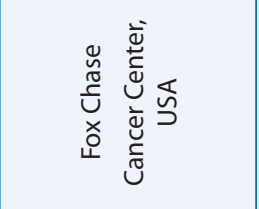 & & 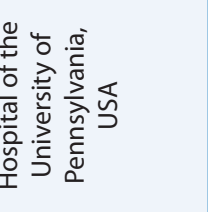 & 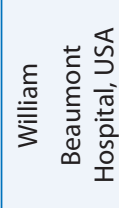 & 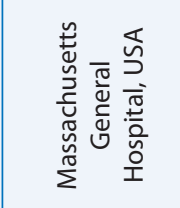 \\
\hline 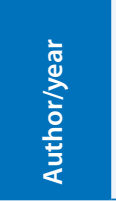 & 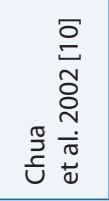 & & 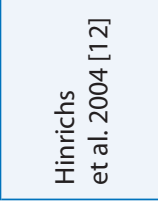 & 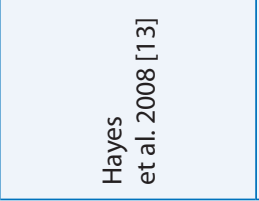 & & 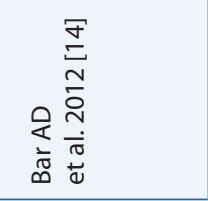 & 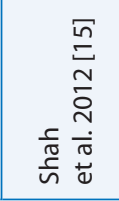 & 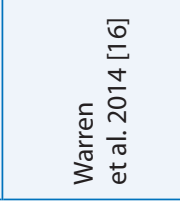 \\
\hline
\end{tabular}




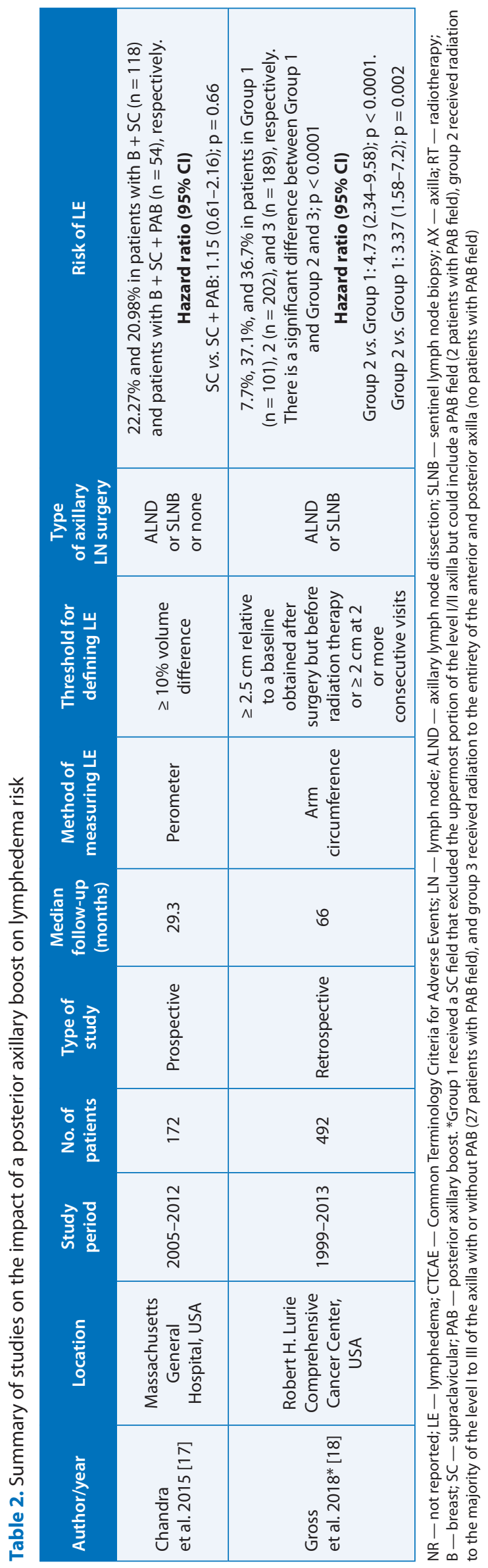

$\mathrm{cm}(2.1-7 \mathrm{~cm}), 4.9 \mathrm{~cm}(1-12 \mathrm{~cm}), 5.1 \mathrm{~cm}(2.5-11.6$ $\mathrm{cm})$, and $3.2 \mathrm{~cm}(1.9-7.4 \mathrm{~cm})$, respectively. In addition, these LNs were positioned well anterior to the midline. In fact, none of these nodes were posterior to the midline. Meanwhile, the dosimetric coverage of the SC and axillary LNs with standard breast tangents and nodal fields was assessed. As reported by Goodman et al., with the use of the standard radiation tangents $90 \%$ of the level I axillary LNs and up to $70 \%$ of level II axillary LNs received 95\% of the prescribed dose to the breast [8]. The results showed that there was a considerable variation in the nodal groups present in the PAB field. In 6/55 patients were no nodal groups were observed in the PAB field [8]. Although the results of the study by Goodman et al. [8] are in good agreement with a previous study by Bentel and colleagues [1], it should be kept in mind that the level I axillary LNs rarely were in the anterior field in the Goodman et al. study [8]. Consistent with their findings, Pierce et al. reported that the level I axillary LNs were in the tangential fields and the apex of the axilla (the infraclavicular region) was treated through the SC field. However, the level II axillary LNs were partially covered in the SC field, and were often supplemented by a PAB field [19].

The PAB field provides a good coverage of the level I/II axillary LNs because these nodes are usually at a greater depth. Of note, in most of the patients, the $\mathrm{PAB}$ field results in a large high-dose region in the AP region of the axilla $[2,7,9]$. This is the most important drawback of this technique, as reported in several studies. To reduce and optimize excessive hot spots at the exit of the PAB field, several techniques have been introduced [2, $7,9]$. The core concept of these techniques was to compensate the overlap with the PAB port. Partial transmission blocks (PTBs) were used to attenuate the anterior field. Rajasekar et al. reported that there was $14-25 \%$ dose variation in the axilla when irradiated by an anterior field and a PAB field [7]. They suggested that using an appropriate PTB in the anterior SC field corresponding to the diverging $\mathrm{PAB}$ field can optimize these dose variations, with the fact that the axillary LNs are deeper than the SC LNs [7]. In another method, a customized compensator was applied to minimize undesirable hot spots in the anterior region of the axilla [9]. Jephcott and colleagues showed that the use of an anterior field with PAB field for treatment of the SC and axil- 
lary LNs in adjuvant RT can provide a good PTV coverage, but it produced an excessive hot spot (> $120 \%)$ in $90 \%$ of patients ( $9 / 10$ patients) [9]. In an attempt to reduce hot spots, an anterior field with $\mathrm{PAB}$ field to the axilla with customized compensation of the AP beam (APcomp-PAB) was assessed. Using this new technique significantly reduced hot spots $>120 \%$ in all cases. Only in one case, was this new technique rejected owing to a high brachial plexus point dose. Also, the APcomp-PAB gave an adequate PTV coverage similar to an anterior field with PAB field [9]. However, the creation of PTBs and compensators is time-consuming and requires a mold room. Nowadays, three-dimensional (3D) printing technology can fabricate treatment and patient-specific devices [20]. Therefore, above-mentioned accessories can be generated by $3 \mathrm{D}$ printing technology that results in reducing time and cost. However, the addition of compensators and PTBs can lead to complexity in treatment planning. Above-mentioned techniques also increase the scatter dose received by the contralateral breast, and require an interruption in daily treatment fraction. It should be noted, however, that toxicity of increased scatter is not clinically relevant, especially since most compensation is electronic these days. Taken all together, these techniques are impractical for routine clinical use.

In an attempt to eliminate these drawbacks, Hernandez et al. have introduced an optimized PAB technique [2]. This technique was mainly based on the PAB technique, but the anterior field was split into two fields: a standard anterior field and another anterior field with the same gantry angle that shielded the region of overlap with the $\mathrm{PAB}$ field to decrease the hot spots. The optimized PAB technique provided adequate coverage of the SC and axillary LNs while minimizing radiation dose to the surrounding normal tissues. Furthermore, this technique overcame the main disadvantage of the $\mathrm{PAB}$ technique, i.e., it reduced hot spots. The mean hot spot dose was $107.7 \%$ in the optimized $\mathrm{PAB}$ technique and $113.5 \%$ in the $\mathrm{PAB}$ technique $(\mathrm{p}=0.006)[2]$.

Recent studies have indicated that the PAB field is a suboptimal technique for treating SC and axillary LNs and new techniques such as intensity modulated RT (IMRT) can provide the opportunity to optimize dose coverage and reduce radiation-induced damage to the surrounding healthy tissues $[3,4]$. The results of the study by Wang et al. showed that CT-based techniques such as an SC field with an anterior boost field and IMRT resulted in superior dose coverage compared with $\mathrm{PAB}$ field [3]. In addition, superior lung sparing and dose conformity to the target were achieved by the IMRT technique. Authors also indicated that the use of the anterior axillary boost (AAB) field resulted in producing smaller hot spots (i.e., $105 \%$ isodose) compared with the PAB field [3]. The mean the treatment volume receiving 105\% or greater of the prescribed dose (V105\%) was $55.9 \mathrm{cc}$ for an $\mathrm{AAB}$ field and $70.0 \mathrm{cc}$ for the PAB technique ( $p=0.037)$. Of note, the PAB technique was not based on CT data, whereas the $A A B$ technique was a CT-based treatment planning. In contrast, when both techniques were based on individual patient's anatomy, the $\mathrm{AAB}$ technique generated higher and larger hot spots; therefore, it is dosimetrically inferior compared to the PAB field, as reported by Hernandez and colleagues [2]. In another study, Sethi et al. compared several 3D techniques and IMRT for level III axillary LNs and SC LNs coverage [4]. Prone IMRT technique resulted in adequate nodal PTV coverage, and reduced ipsilateral lung V20. The results of that study revealed that a supine four-field $3 \mathrm{D}$ conformal RT (3DCRT) using PAB resulted in an average of only $59 \%$ of the LN-PTV receiving $100 \%$ of the prescribed dose [4]. Bentel et al. thought that covering LNs with $90 \%$ of the dose was good enough to justify omitting PAB [1]. In the study by Sethi et al., the PAB technique was criticized for covering only $59 \%$ of the PTV with $100 \%$ of the dose [4]. Coverage by $100 \%$ of the prescription dose is nonstandard.

\section{Impact of posterior axillary boost field on lymphedema}

For breast cancer survivors, lymphedema is considered as one of the most important, physically and emotionally, morbidities after surgery, RT, and taxane-based chemotherapy. It has been globally demonstrated that lymphedema has a significant negative impact on patients' QOL $[5,6]$. Recently, several studies have been conducted to determine independent predictors of lymphedema after breast RT $[15,16,21]$. A PAB field is considered as a potential RT risk factor for lymphedema. To date, several studies have been conducted to quantify 
whether the addition of a PAB field can increase the risk of lymphedema, as outlined in Table 2 [10-18].

It has been shown in some studies that patients with a PAB field have an increased risk of lymphedema $[11,12,14]$. Researchers from Massachusetts General Hospital retrospectively evaluated the risk factors of lymphedema in 727 stage I-II breast cancer patients treated with breast conservation therapy [11]. The 10-year actuarial incidence of lymphedema was $4.1 \%$. Their data suggest that regional node irradiation (RNI) is the only significant risk factor for lymphedema, with a 10-year risk of lymphedema from breast-only RT of $1.8 \% v s .8 .9 \%$ for RNI ( $\mathrm{p}=0.001)$. In that study, the subgroup receiving a SC field alone (three-field technique) was small. Therefore, these patients were grouped with $\mathrm{SC}+\mathrm{PAB}$ field patients (four-field technique). Because most patients were treated with the four-field technique, it is difficult to estimate accurately whether the addition of a PAB field increased the risk of lymphedema compared with a SC field alone [11]. A study from of Roswell Park has revealed that the addition of a PAB field increased lymphedema risk [12]. Hinrichs et al. investigated predictors of lymphedema secondary to post-mastectomy RT in 105 patients [12]. They found that the addition of a PAB field doubled the rate of lymphedema from nearly $23 \%$ to $47 \%(\mathrm{p}=0.047)$. Moreover, their data indicated that RT dose, overlapping the RT technique, RT before 1999, and RT at Roswell Park also were significant predictors of lymphedema. Of note, although SC, internal mammary, mastectomy scar boost, and chest wall tangential photon beam radiation increased the risk of lymphedema, these differences were not statistically significant [12]. In a retrospective study, Bar Ad et al. evaluated the risk factors for progression of lymphedema after breast cancer conversation therapy in 266 stage I-II breast cancer patients at the Hospital of the University of Pennsylvania [14]. The results of univariate Cox models indicated that treatment of the SC nodal region $(\mathrm{p}=0.035)$ and the use of PAB $(\mathrm{p}=0.014)$ were remarkable risk factors for progression of lymphedema. There was no statistically significant difference in arm lymphedema progression between the patients treated with $\mathrm{B}+\mathrm{SC}$ and B-only $(\mathrm{p}=0.4)$, but patients treated with $\mathrm{B}+\mathrm{SC}+\mathrm{PAB}$ were in greater risk of lymphedema, as compared with patients treated with B-only irradiation $(\mathrm{p}=0.01)$ [14]. The freedom from progression at 5 years of follow-up was $73 \%$ for patients treated with B-only irradiation and $36 \%$ for patients receiving $\mathrm{RT}$ to $\mathrm{B}+\mathrm{SC}+\mathrm{PAB}[4]$

In contrast, Chua et al. have compared the lymphedema rates between SC-only RT and $\mathrm{SC}+\mathrm{PAB} \mathrm{RT}$, reporting no significant difference in the rate of lymphedema [10]. From their data it can be seen that the rate of lymphedema was $40 \%$ (6 of 15 patients) in the SC field alone, and 31\% (42 of 136 patients) in the SC + PAB field group. It should be noted that 10 of 136 patients who experienced severe lymphedema were in the SC + PAB group, no severe lymphedema was found in the SC-only group [10] Hayes et al. quantified well the risk of lymphedema from RNI in a series of 2,579 breast cancer patients [13]. In their study, patients were treated with three different radiation fields setting, 2,169 patients $(84 \%)$ received radiation to the breast (B), 226 patients (8.8\%) to the breast and SC LNs $(B+S C)$, and 184 patients to the breast, SC LNs and a PAB (B + SC + PAB). Overall, lymphedema was observed in $18 \%$ of patients at median follow-up of 81 months. The risk of lymphedema was $16 \%, 23 \%$, and $31 \%$ in the B-only, B + SC, and B + SC + PAB group, respectively. The rate of lymphedema in the $\mathrm{B}+\mathrm{SC}+\mathrm{PAB}$ group was comparable with the $47 \%$ incidence of lymphedema found by Hinrichs et al. with the addition of a PAB. In addition, the results for the $\mathrm{N} 1$ subgroup showed that the adding a PAB over tangents led to an increase in the risk of lymphedema ( $p=0.0017)$, but there was no statistically significant increase in lymphedema risk by adding a PAB to B + SC RT ( $p=0.8002)$. In the N2 subgroup, the addition of a $\mathrm{PAB}$ increased the risk of lymphedema 4.5 fold over B + SC RT ( $\mathrm{p}=0.0011)$. Meanwhile, there was no statistically significant difference in the breakdown of nodal failures among the three groups $(\mathrm{p}=0.35)$ [13]. Although the study by Hayes et al. had several main strengths, such as the large sample size and the systematic analysis of all potential lymphedema risk factors, retrospective nature of the study and extensive LN dissection or the number of positive LNs can be great risk factors for developing lymphedema owing to the interference of lymphatic drainage irrespective of RT field arrangement.

A study from William Beaumont Hospital evaluated the rates of breast cancer-related lymphedema in 1497 patients treated with whole-breast irradiation [15]. RT parameters were analyzed in patients 
with and without lymphedema. The data from that study indicated that the addition of regional irradiation (i.e., SC, PAB, and internal mammary field) over whole breast irradiation did not statistically significantly increase the incidence of lymphedema. The findings showed that adding a PAB field approximately doubled the actuarial rate of lymphedema, but it was no statistically significant (14.7\% with $\mathrm{PAB} v$ s. $7.3 \%$ without $\mathrm{PAB}, \mathrm{p}=0.10)$ [15].

Consistent with Hayes et al. study [13], in a prospective single-institution cohort study, Warren et al. determined that the addition of a PAB to SC field did not increase the risk of lymphedema in comparison with SC alone [16]. No statistically significant difference in lymphedema risk between $\mathrm{SC}$ and SC + PAB was observed $(\mathrm{p}=0.96)$. These findings also were in good agreement with a prior study by Chua et al. [10]. However, Warren et al. found that the addition of RLN radiation, regardless of radiation field type (i.e. SC or SC + PAB), compared with breast/chest wall radiation alone significantly increased the risk of lymphedema with a hazard ratio of $1.7(\mathrm{p}=0.025)$ [16]. Consistent with Warren et al. study, Graham et al. also reported that using axillary irradiation results in a significant increase in the lymphedema rates whether defined by using a PAB field $(\mathrm{p}=0.004)$ or subdivided by any irradiation lateral to the coracoid $(p=0.002)$ [22]. In another report, dosimetric risk factors for the incidence of lymphedema in 172 breast cancer patients treated with RNI radiation at the Massachusetts General Hospital were analyzed [17]. The 2-year cumulative incidence of lymphedema was $22.27 \%$ and $20.98 \%$ for SC and $\mathrm{SC}+\mathrm{PAB}$, respectively. Meanwhile, RLN radiation field type was not associated with the development of lymphedema ( $\mathrm{p}=0.66$ for $\mathrm{SC} v$ s. $\mathrm{SC}+\mathrm{PAB}$ field). In addition, there was no relationship either between lymphedema risk and other specific RT parameters such as fraction size, extent of humeral head, beam energy, and breast tangent type [17]. It should be noted that the vast majority of patients with lymphedema received axillary LN dissection (87\%) [17], a well-documented risk factor for lymphedema development [23]. This, along with a relatively small sample size $(\mathrm{n}=172)$ can obscure the impact of the independent RT risk factor on the development of lymphedema.

As reported by Hayes and colleagues, in patients with 4-9 positive LNs (N2 subgroup, $\mathrm{n}=109$ ) that most often undergo RNI, although the SC field did not increase the risk of lymphedema in comparison with tangents, the use of a PAB field increased lymphedema risk 4.5 fold over tangents and a SC [13]. In this subgroup of patients, this strong correlation resulted in a fall of the number of LNs dissected [13]. As a result, it is necessary to avoid a PAB unless there are absolute indications for its application. It is clear that the rate of nodal recurrence in the $\mathrm{PAB}$ group is lower than in the B-only or B + SC group (18/2169 patients in the B group, $8 / 226$ patients in the $B+S C$ group, and 2/184 patients in the $\mathrm{B}+\mathrm{SC}+\mathrm{PAB}$ group) [13]. However, studies have not demonstrated the advantage of a PAB radiation in improving nodal control. Of note, one possible reason for decreasing nodal recurrence in the PAB group may be associated with developed metastatic disease in these patients that results in ceasing time surveillance for nodal recurrences.

As mentioned above, previous reports investigated differences in radiation beam arrangement, i.e., adding a PAB field, but did not clearly determine the volume of axillary tissue irradiated. On the other hand, field designs may be more important for the incidence of lymphedema than field arrangement [18]. A recent study by Gross et al. has documented the relationship between RT field design and lymphedema development [18]. A cohort of 492 patients with stage II-IV breast cancer who had received RLN radiation following breast surgery were divided into three subgroups according to different radiation fields. Group $1(\mathrm{n}=101)$ received a SC field that excluded the uppermost portion of the level I/II axilla but could include a PAB field, group $2(n=202)$ received radiation to the majority of the level I to III of the axilla with or without $\mathrm{PAB}$, and group $3(\mathrm{n}=189)$ received radiation to the entirety of the anterior and posterior axilla. On the other hand, no patients in group 3 treated were with a $\mathrm{PAB}$ field, while more patients in group 2 received a $\mathrm{PAB}$ field when compared with group 1 , as the control group. The 5-year lymphedema rates in group $2(37.1 \%)$ and group 3 (36.7\%) were significantly higher than those in group $1(7.7 \%$, $\mathrm{p}<0.0001$ ), despite the fact that more of these patients had undergone sentinel LN biopsy (15-19\% vs. $6 \%$ ) when compared with patients in group 1 . Of note, the likelihood of the development of lymphedema after sentinel LN biopsy is lower than axil- 
lary LN dissection. The incidence of lymphedema at 12 months was $11 \%$ after axillary LN dissection and $6 \%$ after sentinel LN biopsy in the American College of Surgeons Oncology Group Trial Z0011 [24]. Gross et al. suggest that the lateral border of the $2 \mathrm{D}$ nodal $\mathrm{RT}$, as a proxy for the volume of tissue irradiated, is a significant factor for lymphedema development [18]. In other words, radiation of the upper level I to II axilla appears to be particularly important for the development of lymphedema risk after axillary dissection.

As observable in above-mentioned studies, the impact of a PAB RT on the development of lymphedema is controversial. A possible source of these controversies can be the fact that it was unknown what degree of the upper axilla was treated with RNI. As described by Gross et al., radiation field design can result in increasing radiation to the volume of axillary tissues, thereby increasing the incidence of lymphedema [18], whereas other previous studies focused on radiation field arrangement. Besides, a recent study has identified that dose delivered to the axillary-lateral thoracic vessel juncture (ALTJ, the area superior to axillary level I) can be associated with lymphedema risk [25]. As a consequence, radiation field design can be a more relevant risk factor for development of lymphedema than radiation field arrangement. Another issue of possible relevance for the impact of a PAB field on the incidence of lymphedema is the lack of uniformity in measuring lymphedema. As outlined in Table 2, investigators have used multiple metrics to quantify and qualify the incidence of lymphedema, including perometry, arm circumference, etc. Therefore, standardizing the method of detecting lymphedema is needed. The testing of the inclusion of upper arm lymphatics in the regional LN irradiation target volume, and universal methodology measuring lymphedema are all areas for possible future studies. Besides, as observable in Table 2, most studies were retrospective and, therefore, may suffer from underestimating actual incidence of lymphedema owing to the lack of documentation in medical record, delayed onset, etc. Moreover, retrospective studies have higher potential sources of bias and confounding. These are issues that will require additional investigation before definitive recommendations can be made regarding the therapeutic index of a $\mathrm{PAB}$ radiation.

\section{Conclusion}

There are few studies that have investigated whether a PAB is necessary for RLNI. A key factor in using a PAB is the depth of axillary LNs that today is well determined on the basis of CT. The results of this literature review show that depending on dose distribution and patient's anatomy, a PAB field is employed to supplement axillary dose. The $\mathrm{PAB}$ field provides a good coverage of level I/II axillary LNs because these LNs are located much deeper. The most important concern regarding a PAB is that it produces a large high-dose region in the AP region of the axilla. To reduce drawbacks of a PAB field RT, physicists and radiation oncologists should optimize and develop a traditional PAB field, as suggested by Jephcott et al. [9] and Hernandez et al. [2]. IMRT technique can also provide excellent dose coverage and simultaneously reduce radiation-induced normal tissue toxicity. However, the use of IMRT techniques involves higher complexity in treatment planning and delivery steps and, therefore, they are not commonly used for the SC and axillary LNs irradiation. Moreover, prospective trials are needed to clarify the efficacy of this approach.

On the one hand, a recent study has suggested that volume and distribution of axillary irradiation may be the most important risk factors of lymphedema development compared with beam arrangement alone. On the other hand, in studies where beam arrangement was investigated, there are conflicting reports on the association of a $\mathrm{PAB}$ field with the incidence of lymphedema. Although data reveal that the addition of RNI to breast irradiation significantly increases the risk of lymphedema in patients with breast cancer, prospective studies and the vast majority of retrospective studies have reported the use of a PAB RT does not result in a statistically significant increase in the risk of lymphedema development over SC-alone $\mathrm{RT}$. Of note, there is a trend of increasing risk of lymphedema with a PAB field. Therefore, clinicians should continue to weigh lymphedema risk in individual patients against the benefit of a $\mathrm{PAB}$, in particular after axillary dissection. In the light of these findings, although definitive recommendations regarding the clinical benefit of a PAB field are relatively difficult, axillary radiation with a $\mathrm{PAB}$ field is recommended for targeting level I/II axilla 
if there are clinically matted LNs, $\geq 4$ involved axillary LNs, gross extranodal spread of tumor, all LNs dissected positive, positive sentinel LNs without a subsequent completion axillary dissection, an inadequate axillary dissection $(<6 \mathrm{LNs})$, or the highest LNs dissected being positive $[3,13]$.

\section{Conflict of interests}

The authors declare that they have no conflicts of interest.

\section{Funding}

Not applicable.

\section{Availability of data and materials}

The datasets used and/or analyzed during the current study are available from the corresponding author on reasonable request.

\section{References}

1. Bentel GC, Marks LB, Hardenbergh PH, et al. Variability of the depth of supraclavicular and axillary lymph nodes in patients with breast cancer: is a posterior axillary boost field necessary? Int J Radiat Oncol Biol Phys. 2000; 47(3): 755-758, doi: 10.1016/s0360-3016(00)00485-5, indexed in Pubmed: 10837961.

2. Hernandez V, Arenas M, Müller K, et al. An optimized posterior axillary boost technique in radiation therapy to supraclavicular and axillary lymph nodes: a comparative study. Med Dosim. 2013; 38(4): 413-417, doi: 10.1016/j. meddos.2013.05.003, indexed in Pubmed: 23910694.

3. Wang X, YU TK, Salehpour M, et al. Breast cancer regional radiation fields for supraclavicular and axillary lymph node treatment: is a posterior axillary boost field technique optimal? Int J Radiat Oncol Biol Phys. 2009; 74(1): 86-91, doi: 10.1016/j.ijrobp.2008.07.016, indexed in Pubmed: 18805650.

4. Sethi RA, No HS, Jozsef G, et al. Comparison of threedimensional versus intensity-modulated radiotherapy techniques to treat breast and axillary level III and supraclavicular nodes in a prone versus supine position. Radiother Oncol. 2012; 102(1): 74-81, doi: 10.1016/j. radonc.2011.09.008, indexed in Pubmed: 21993404.

5. Ahmed RL, Prizment A, Lazovich $D$, et al. Lymphedema and quality of life in breast cancer survivors: the lowa Women's Health Study. J Clin Oncol. 2008; 26(35): 5689-5696, doi: 10.1200/JCO.2008.16.4731, indexed in Pubmed: 19001331.

6. Jäger G, Döller W, Roth R. Quality-of-life and body image impairments in patients with lymphedema. Lymphology. 2006; 39(4): 193-200, indexed in Pubmed: 17319632.

7. Rajasekar D, Datta NR, Das KJ, et al. Partial transmission block for optimization of anterior supraclavicular-posterior axillary boost in the radiation therapy of carcinoma breast. Med Dosim. 1998; 23(2): 105-108, doi: 10.1016/ s0958-3947(98)00005-3, indexed in Pubmed: 9664276.
8. Goodman RL, Grann A, Saracco P, et al. The relationship between radiation fields and regional lymph nodes in carcinoma of the breast. Int J Radiat Oncol Biol Phys. 2001;50(1): 99-105, doi: 10.1016/s0360-3016(00)01581-9, indexed in Pubmed: 11316551.

9. Jephcott CR, Tyldesley S, Swift CL. Regional radiotherapy to axilla and supraclavicular fossa for adjuvant breast treatment: a comparison of four techniques. Int J Radiat Oncol Biol Phys. 2004; 60(1): 103-110, doi: 10.1016/j. ijrobp.2004.02.057, indexed in Pubmed: 15337545.

10. Chua B, Ung O, Boyages J. Competing considerations in regional nodal treatment for early breast cancer. Breast J. 2002; 8(1): 15-22, doi: 10.1046/j.1524-4741.2002.08004.x, indexed in Pubmed: 11856156.

11. Coen JJ, Taghian AG, Kachnic LA, et al. Risk of lymphedema after regional nodal irradiation with breast conservation therapy. Int J Radiat Oncol Biol Phys. 2003; 55(5): 1209-1215, doi: 10.1016/s0360-3016(02)04273-6, indexed in Pubmed: 12654429.

12. Hinrichs CS, Watroba NL, Rezaishiraz H, et al. Lymphedema secondary to postmastectomy radiation: incidence and risk factors. Ann Surg Oncol. 2004; 11(6): 573-580, doi: 10.1245/ASO.2004.04.017, indexed in Pubmed: 15172932.

13. Hayes SB, Freedman GM, Li T, et al. Does axillary boost increase lymphedema compared with supraclavicular radiation alone after breast conservation? Int J Radiat Oncol Biol Phys. 2008; 72(5): 1449-1455, doi: 10.1016/j. ijrobp.2008.02.080, indexed in Pubmed: 19028274.

14. Bar Ad V, Dutta PR, Solin LJ, et al. Time-course of arm lymphedema and potential risk factors for progression of lymphedema after breast conservation treatment for early stage breast cancer. Breast J. 2012; 18(3): 219-225, doi: 10.1111/j.1524-4741.2012.01229.x, indexed in Pubmed: 22487094.

15. Shah C, Wilkinson JB, Baschnagel A, et al. Factors associated with the development of breast cancer-related lymphedema after whole-breast irradiation. Int J Radiat Oncol Biol Phys. 2012; 83(4): 1095-1100, doi: 10.1016/j. ijrobp.2011.09.058, indexed in Pubmed: 22099041.

16. Warren LEG, Miller CL, Horick N, et al. The impact of radiation therapy on the risk of lymphedema after treatment for breast cancer: a prospective cohort study. Int J Radiat Oncol Biol Phys. 2014; 88(3): 565-571, doi: 10.1016/j. ijrobp.2013.11.232, indexed in Pubmed: 24411624.

17. Chandra RA, Miller CL, Skolny MN, et al. Radiation therapy risk factors for development of lymphedema in patients treated with regional lymph node irradiation for breast cancer. Int J Radiat Oncol Biol Phys. 2015; 91(4): 760-764, doi: 10.1016/j.ijrobp.2014.12.029, indexed in Pubmed: 25752389.

18. Gross JP, Sachdev S, Helenowski IB, et al. Radiation Therapy Field Design and Lymphedema Risk After Regional Nodal Irradiation for Breast Cancer. Int J Radiat Oncol Biol Phys. 2018; 102(1): 71-78, doi: 10.1016/j.jirobp.2018.03.046, indexed in Pubmed: 30102206.

19. Pierce LJ, Oberman HA, Strawderman MH, et al. Microscopic extracapsular extension in the axilla: is this an indication for axillary radiotherapy? Int J Radiat Oncol Biol Phys. 1995; 33(2): 253-259, doi: 10.1016/03603016(95)00081-9, indexed in Pubmed: 7673012.

20. Zhao Y, Moran K, Yewondwossen M, et al. Clinical applications of 3-dimensional printing in radiation therapy. 
Med Dosim. 2017; 42(2): 150-155, doi: 10.1016/j.meddos.2017.03.001, indexed in Pubmed: 28495033.

21. Shaitelman SF, Chiang YJ, Griffin KD, et al. Radiation therapy targets and the risk of breast cancer-related lymphedema: a systematic review and network meta-analysis. Breast Cancer Res Treat. 2017; 162(2): 201-215, doi: 10.1007/ s10549-016-4089-0, indexed in Pubmed: 28012086.

22. Graham P, Jagavkar R, Browne L, et al. Supraclavicular radiotherapy must be limited laterally by the coracoid to avoid significant adjuvant breast nodal radiotherapy lymphoedema risk. Australas Radiol. 2006; 50(6):578-582, doi: 10.1111/j.1440-1673.2006.01658.x, indexed in Pubmed: 17107530.

23. Veronesi U, Paganelli G, Viale G, et al. A randomized comparison of sentinel-node biopsy with routine axil- lary dissection in breast cancer. N Engl J Med. 2003; 349(6): 546-553, doi: 10.1056/NEJMoa012782, indexed in Pubmed: 12904519.

24. Lucci A, McCall LM, Beitsch PD, et al. American College of Surgeons Oncology Group. Surgical complications associated with sentinel lymph node dissection (SLND) plus axillary lymph node dissection compared with SLND alone in the American College of Surgeons Oncology Group Trial Z0011. J Clin Oncol. 2007; 25(24):3657-3663, doi: 10.1200/ JCO.2006.07.4062, indexed in Pubmed: 17485711.

25. Gross JP, Lynch CM, Flores AM, et al. Determining the Organ at Risk for Lymphedema After Regional Nodal Irradiation in Breast Cancer. Int J Radiat Oncol Biol Phys. 2019; 105(3):649-658, doi: 10.1016/j.ijrobp.2019.06.2509, indexed in Pubmed: 31260718 